\title{
Heterotopic trigeminal pregnancy after in vitro fertilization and embryo transfer: A case report
}

Carlo V Bellieni ${ }^{1}$, Libera Troìa ${ }^{2}$, Michela Torricelli ${ }^{2}$, Matteo Benvenuti ${ }^{3}$, Giuseppe Buonocore ${ }^{1}$ and Filiberto Maria Severi $^{2 *}$

${ }^{1}$ Neonatal Intensive Care Uniti, University Hospital, Siena, Italy

${ }^{2}$ Obstetrics and Gynecology, Department of Molecular and Developmental Medicine, University of Siena, Siena, Italy

${ }^{3}$ Department of Legal Medicine, University of Siena, Italy

A caucasic 42-year-old woman, first pregnancy, with a diagnosis of female factor infertility caused by ovulation failure and azoospermia in the male partner, underwent an IVF procedure using sperm and ovocytes donors. The patient had 2 embryos transferred 26 days before she presented to the Obstetric Department with a chief symptom of abdominal pain.

Laboratory testing revealed a serum $\beta$-human chorionic gonadotropin level of $56779 \mathrm{mIU} / \mathrm{mL}$.

A transvaginal pelvic sonographic examination demonstrated a intrauterine twin pregnancy without embryonic structures, a third gestational sac in the right uterine adnexa with an embryo measuring $4,3 \mathrm{~mm}$ length according to $5+5$ weeks of amenorrhea with cardiac activity and the presence of free fluid in the pouch of Douglas.

A bilateral salpingectomy and uterine curettage was performed.

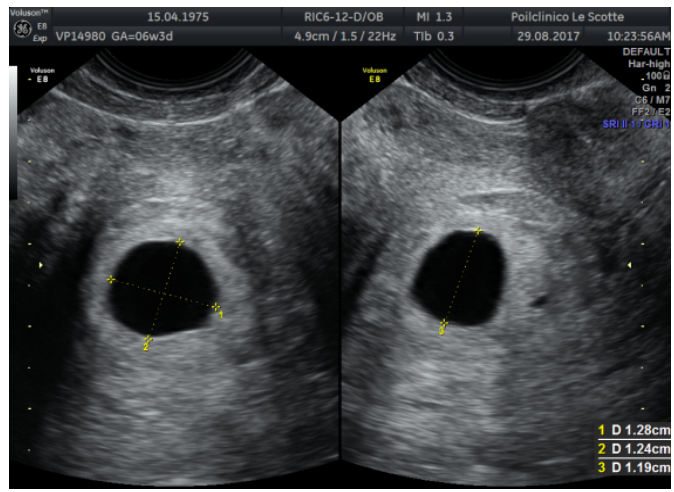

Figure 1. Transvaginal sagittal section showing the first empty intrauterine gestation sac.

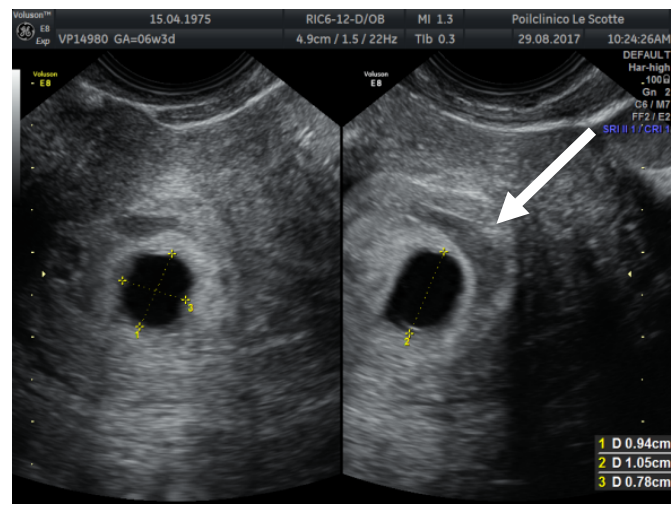

Figure 2. Second empty intrauterine gestation sac, located below the first and smaller dimensions. The arrow indicates an area of amniochorial detachment.

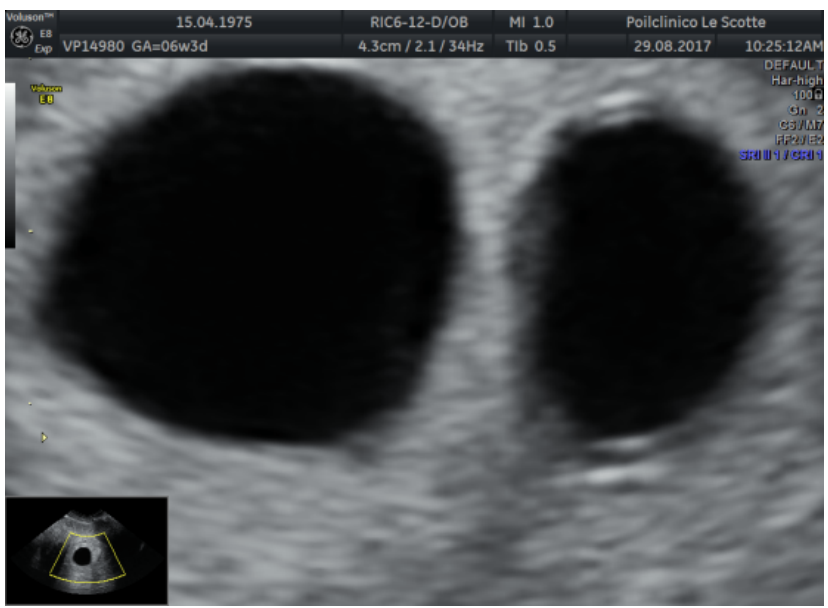

Figure 3. Ultrasonographic find of both intrauterine gestational sacs showing twin pregnancy.

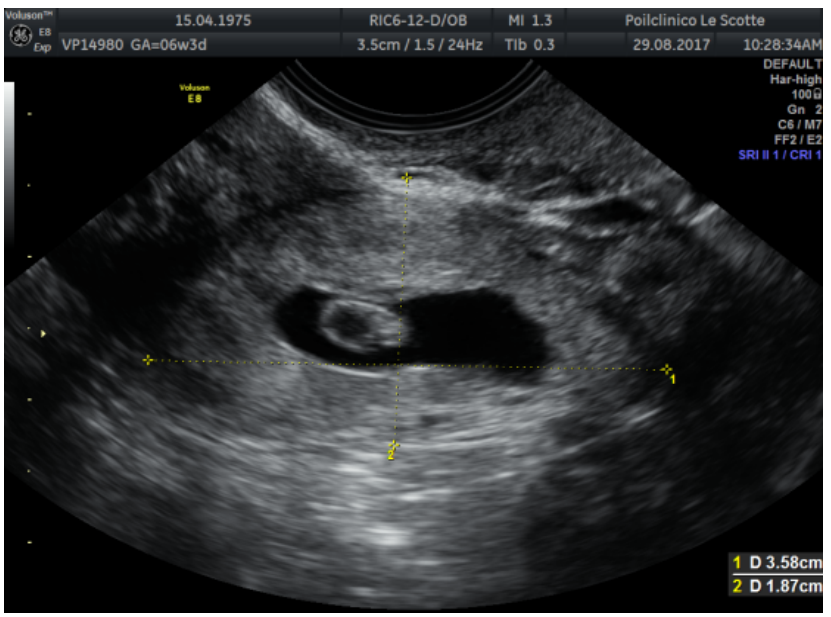

Figure 4. Ultrasound image showing an ectopic pregnancy in the ampullary region of the right fallopian tube with the presence of embryonic structures: gestational sac, yolk sac and fetal pole.

Correspondence to: Filiberto Maria Severi, MD, PhD, Obstetrics \& Gynecology, Department of Molecular and Developmental Medicine, University of Siena Policlinico "Le Scotte" Viale Bracci, 53100 Siena, Italy, Tel: +39 0577 58.6601, E-mail: filiberto.severi@unisi.it

Received: September 02, 2017; Accepted: September 18, 2017; Published: September 21, 2017 


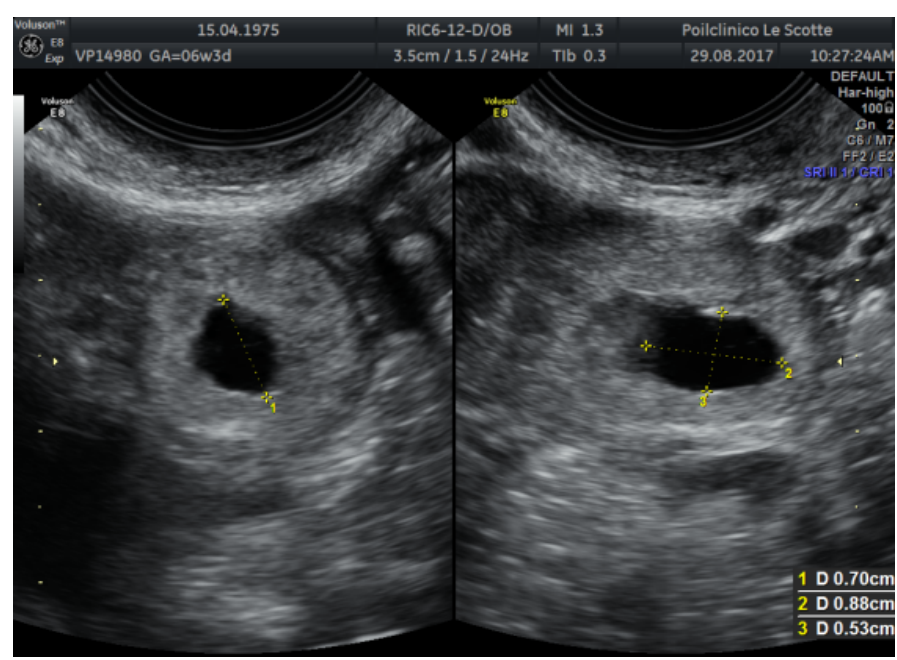

Figure 5. Ultrasonographiv find of the tubal sign, defined by the presence of a round hypoechoic adnexal collection with a well-defined hyperechoic rim. This represents a gestational sac (hypoechoic center) with surrounding trophoblast (hyperechoic rim).

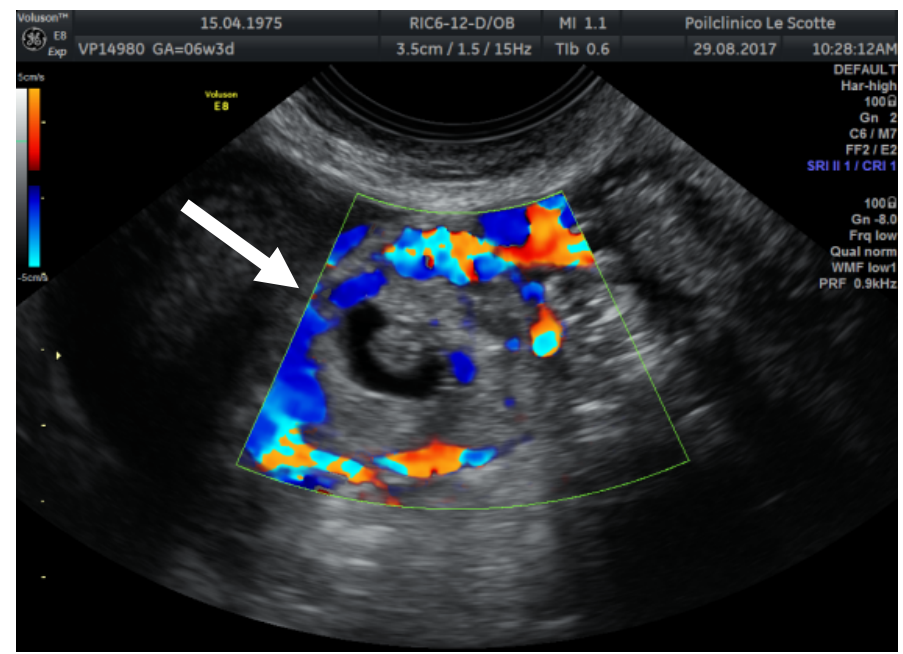

Figure 6. Tubal ectopic pregnancy by transvaginal ultrasound. The arrow indicates the ectopic gestation with circumferential Doppler flow, called the "Ring of Fire".

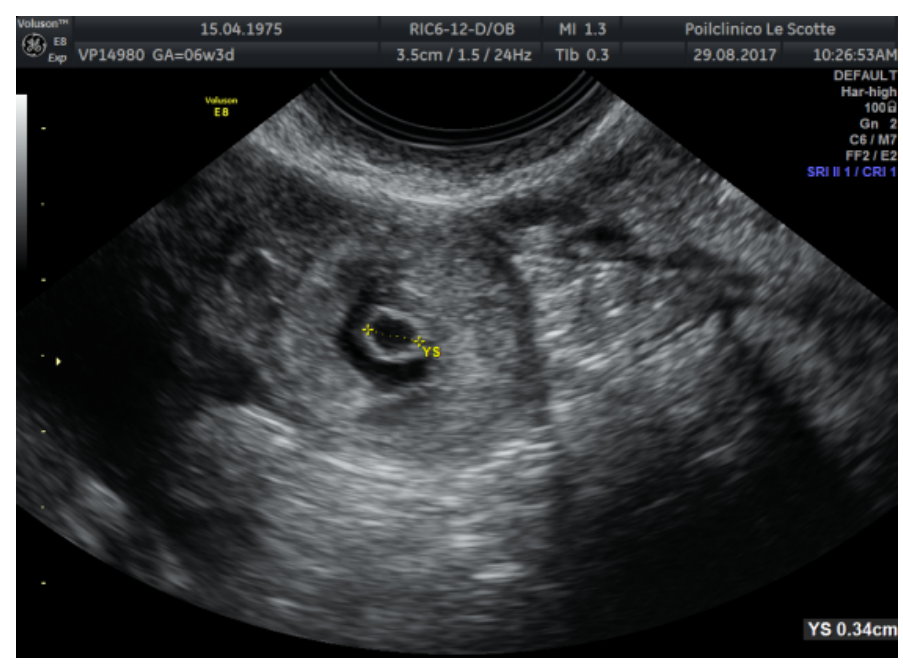

Figure 7. Ultrasound examination revealing the presence of yolk sac of tubal pregnancy.

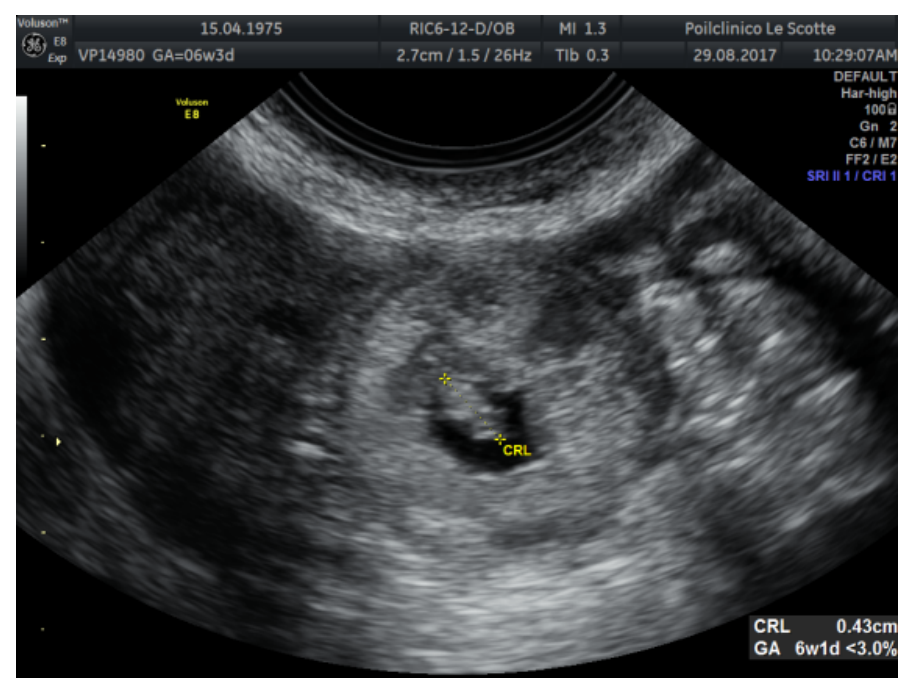

Figure 8. Ultrasound image showing the presence of an embryo with crown-rump length measurements of $4,3 \mathrm{~mm}$, according to $5+5$ weeks of amenorrhea.

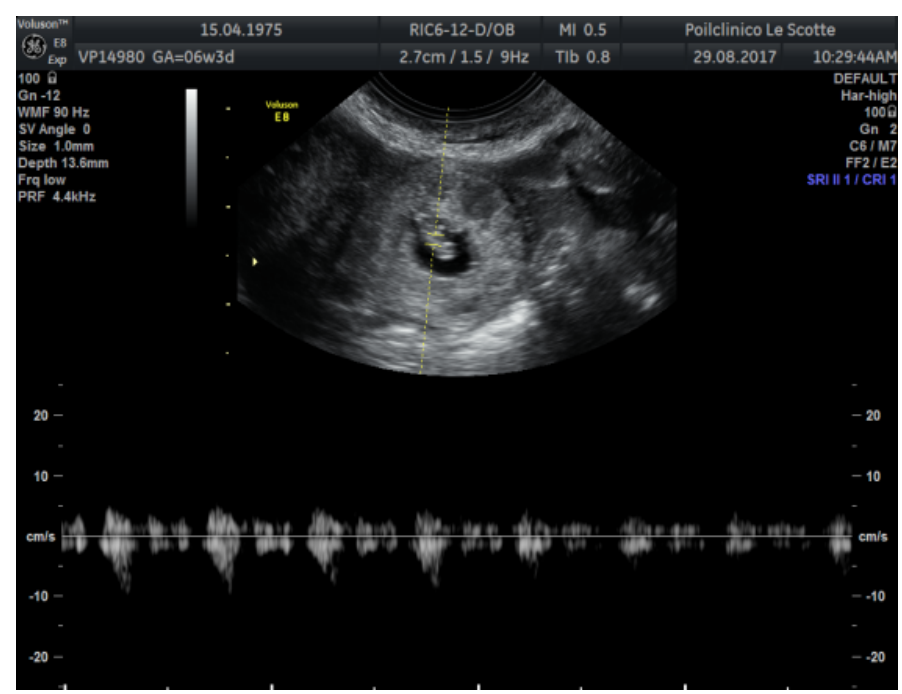

Figure 9. Fetal pole with visible fetal heartbeat detected at Power-Doppler.

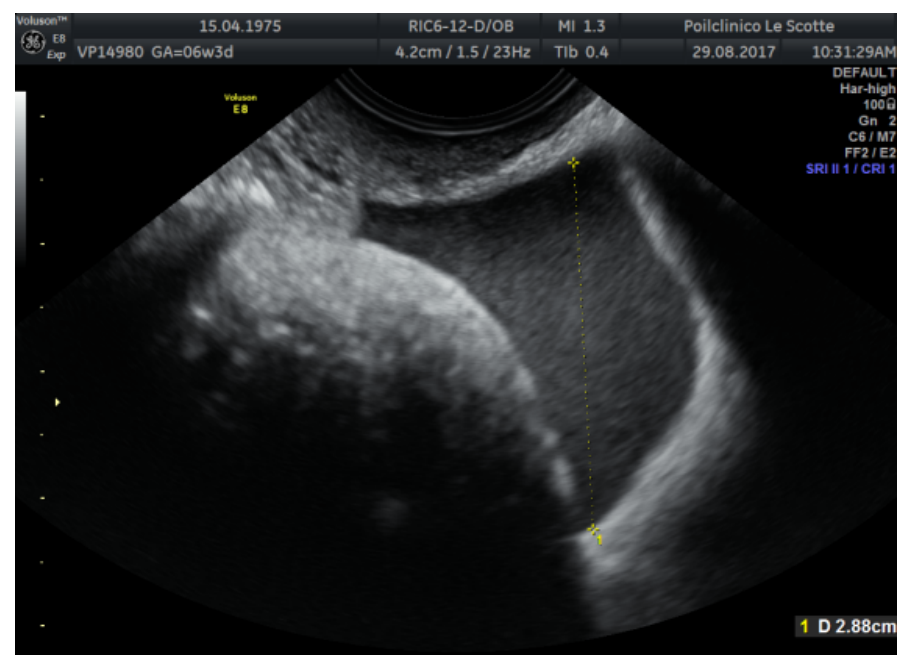

Figure 10. Ultrasonographic find of free fluid in the pouch of Douglas.

Copyright: C2017 Bellieni CV. This is an open-access article distributed under the terms of the Creative Commons Attribution License, which permits unrestricted use, distribution, and reproduction in any medium, provided the original author and source are credited. 